TPI-MINN-99-13

NUC-MINN-99/5-T

UMN-TH-1749

March 1999

\title{
Initial Conditions for Parton Cascades ${ }^{1}$
}

\author{
Larry McLerran \\ Theoretical Physics Institute, University of Minnesota, Minneapolis, MN 55455
}

\begin{abstract}
Abstract: I discuss the initial conditions for a parton cascade.
\end{abstract}
\section{INTRODUCTION}

Klaus Kinder-Geiger was a postdoctoral fellow with us at the University of Minnesota from 1991-1993.

I remember well the first seminar he gave to us on the work he had been doing with Berndt Muller concerning the parton cascade model. I was very excited by what he was doing, and I was asking him question after question. This was at a time at the University of Minnesota recently after we had hired a number of Russians, so 2 hour seminars were not unusual. (We no longer have such long seminars. The Russians are real Americans now.) Klaus was needless to say nervous about the time he was taking, and Sharon, who was in the audience, was I think more than a little bothered by what she perceived as my harassment of Klaus.

I don't think Klaus fully understood how much I respected him after that talk. He was one of the few young people I knew who were not afraid to say they didn't understand something, who was excited about exploring new ways of thinking, and most important, had a deep understanding of what it was he was doing.

I guess we all knew Klaus as an unconventional thinker. When he was with us, he had the typical fears and lack of confidence of all people his age. The following story illustrates this: We were discussing hiring new postdocs at Minnesota and Joe Kapusta and I invited Klaus to come to the meeting and join in the discussion. The first thing I did was go down the list of people, and anyone who had published less than three papers a year since they got their Ph. D., I refused to further consider.

1) Talk presented at RHIC Physics and Beyond: Kay Kay Gee Day, Brookhaven National Laboratory, Upton, Long Island NY, Oct. 1998 
Klaus muttered something about how this wasn't really very fair, and I answered back that I didn't want to hire lazy people. Klaus had been with us for about 6 months at this time and had submitted a paper, maybe two, for publication. In the next few months, he submitted about half a dozen.

Klaus was the most prolific postdoc which we ever hired in nuclear theory at the University of Minnesota. His papers were not superficial and each involved much work and thinking.

Klaus and I talked much but never worked together. He was captured by my colleague Joe Kapusta. Klaus had a profound impact on my thinking nevertheless. He got me very interested in his picture of the very early stages of heavy ion collisions, and this is the subject of this talk.

To understand the parton cascade model, [1]- [2] one needs a space-time picture of nucleus-nucleus collisions. Such a space-time picture was developed by Bjorken and I shall summarize it in this introduction. [3] We concentrate on the central region of collisions at asymptotically high energy. We assume that the rapidity density of produced particle is slowly varying, slow enough so that we can treat the distribution

$$
\frac{d N}{d y}=\text { contant }
$$

If this is the case, the space-time dynamics for particle produced in the central region should be longitudinally Lorentz boost invariant. This means that the dynamical evolution of the particles produced in the collision is described by only on parameter $\tau=\sqrt{t^{2}-z^{2}}$. We also will assume that the transverse size of the system is large enough so that one can ignore effects such as the transverse expansion of the system. The other longitudinal variable is the space-time rapidity

$$
\eta=\frac{1}{2} \ln \left(\frac{t+z}{t-z}\right)
$$

which under a longitudinal Lorentz boost changes by a constant.

Note that for a free streaming particle, $\eta=y$ since

$$
\eta=\frac{1}{2} \ln \left(\frac{t+z}{t-z}\right)=\frac{1}{2} \ln \left(\frac{1+v_{z}}{1-v_{z}}\right)=\frac{1}{2} \ln \left(\frac{E+p_{z}}{E-p_{z}}\right)=y
$$

We see therefore that

$$
\frac{d N}{d y} \sim \frac{d N}{d \eta}=\frac{d N}{d z} \frac{t}{\tau^{2}}
$$

The particles produced at $z=y=0$ therefore expand and dilute their density as

$$
\frac{d N}{d z}=\frac{\text { constant }}{t}
$$


In an isentropic expansion, as will be the case later in the collision after the particle have thermalize, the entropy density $\sigma$ satisfies

$$
\tau \sigma=\tau_{0} \sigma_{0}
$$

Here $\tau_{0}$ is the initial thermalization time, for which a variety of arguments suggest that $\tau_{0} \leq 1 \mathrm{Fm} / \mathrm{c}$

We expect that the entropy will be approximately conserved as the system expands. If there is a first order phase transition, then there will be some entropy production, but again for the typical time scales characteristic of heavy ion collisions, we do not expect a dramatic increase in the entropy. Of course as the system expands, the degrees of freedom of the system change dramatically. Early on, we expect that the system will be an almost ideal gas of quarks and gluons. Late on the gas is hadronic, and very late it is a gas of far separated almost non-interacting pions. If the hadronic gas decouples when the pions are still to a good approximation massless, as will be the case for decoupling temperatures $T_{\text {decoupling }} \geq 100 \mathrm{MeV}$ (recall the average energy is $3 \mathrm{~T}$, so that $\left(m_{\text {pion }} / E\right)^{2} \sim .1$ ), then one can show that entropy production implies that the number of gluons initially is the same as the number of pions. Therefore the number density of gluons early on is

$$
\frac{N}{V}=\frac{1}{\tau_{0} \pi R^{2}} \frac{d N_{\text {pions }}}{d y}
$$

For the typical rapidity density of pions seen at RHIC, this leads to initial temperatures $T \geq 200 \mathrm{MeV}$. This should be sufficient to produce a quark gluon plasma.

In Fig. 1, a space-time picture of the evolution of matter produced in ultrarelativistic nuclear collisions is shown. After the time $\tau_{0}$ when thermalization occurs, the system expands. At some time and corresponding temperature, the system converts from a quark-gluon plasma into a hadron gas. This may take some time, and go through a mixed phase if there is truly a phase difference between hadronic matter and a quark-gluon plasma. If there is no true phase change, the system nevertheless changes its properties dramatically and to do so involves time. At much later time, the system freezes out and produces free streaming particles.

\section{WHAT HAPPENS BEFORE $\tau_{0}$ ?}

What happens in the time between $\tau=0$ and $\tau_{0}$ ? Surely, the earlier one goes in time, the more energetic are particle interactions. Weak coupling methods should therefore be at their best, and one should be able to compute, at least in the limit of very high energies and very large nuclei, from first principles in QCD.

It is reasonable to assume that the matter when it is first formed in heavy ion collisions is in some sort of non-thermal distribution. The matter must therefore thermalize. Klaus Kinder-Geiger and Berndt Muller made a daring proposal in an 


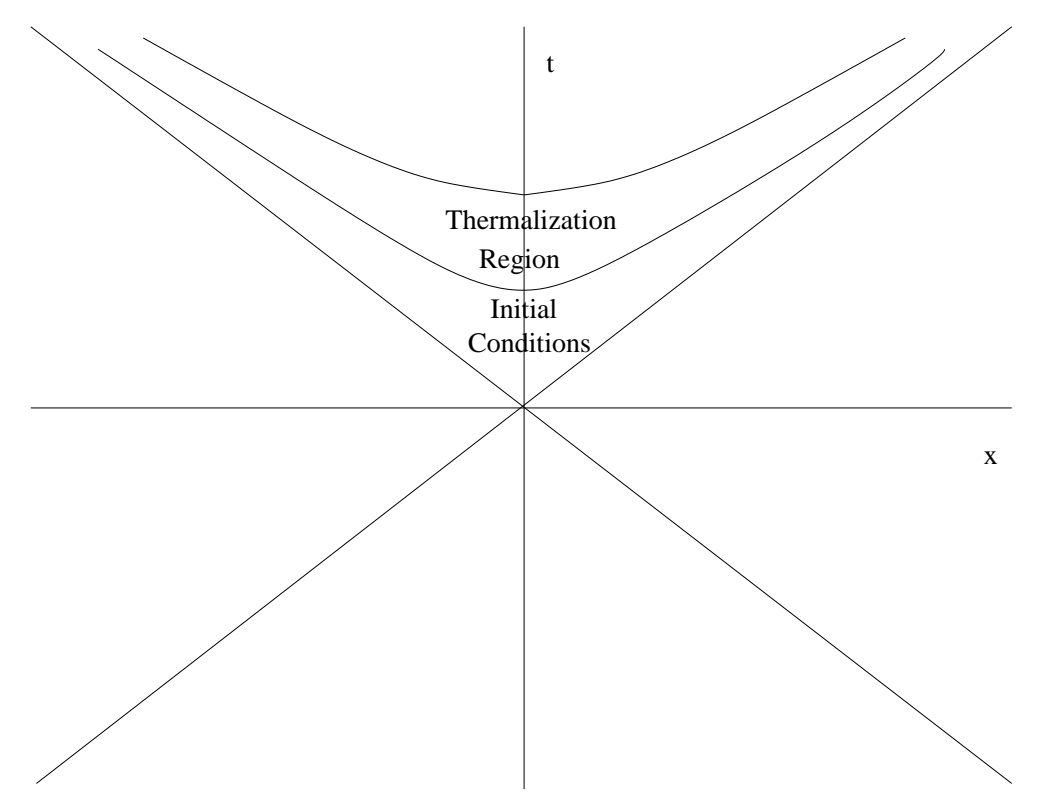

FIGURE 1. A Space-Time Diagram of Ultrarelativistic Nuclear Collisions

attempt to understand the thermalization. They assumed the momentum spacedistribution of partons just after formation was given by the parton distribution functions.

This assumption deserves a little comment since the parton distribution functions specify only the longitudinal momentum space distribution of the partons. Both the transverse momentum structure and the space-time positions of the partons at formation must be assumed. Some guidance about what are reasonable assumptions are given by uncertainty principle arguments, but the coordinate space picture is nevertheless assumed.

In fact the uncertainty principle and quantum mechanics limits the region where the parton cascade can be applied. In order to use a cascade, one must specify the phase space distribution of particles $f(\vec{p}, \vec{x}, t)$. This involves specifying both the position and coordinates of the particles, and is inconsistent with a quantum mechanical description. (One can formally define a phase space distribution function for a fully quantum system, but the distribution will in general lack positivity, and usually will violate it in the region of phase space where the quantum effects are important.) At the earliest times in the collision, the system is described by two quantum mechanical wavefunctions which describe the nuclei. Therefore for some sufficiently early time, the parton cascade description must fail.

One can also see that one must go beyond partons to describe the earliest times in the collisions. At the earliest time, the density of fast moving quarks and gluons is very high. If we use cascade theory to describe their effect on long wavelength quanta such as will be produced in the central region, we will have each of the quanta acting incoherently. This is because in a cascade, only matrix elements 
squared for single particle scattering occur. On the other hand, we know that when we compute the field associated with these quanta, their effect is tempered since because of overall color neutrality for confined particles. Any colored field will therefore be reduced in strength in the infrared, and their effect on long wavelength quanta will be reduced.

Klaus and Berndt tried to phenomenologically include the effect of quantum mechanics and classical charge coherence in two separate ways. The first was to assume that particles were not produced and could interact until after a characteristic formation time took place in the rest frame of the particle. This has the effect of delaying the cascade description until after the formation time has taken place. It evades the question of whether the parton distributions are modified during the time from the initial collision $\tau=0$ until the formation time. During this time, the evolution is quantum mechanical, but in the complicated collision environment, there may be a non-trivial quantum evolution of the distributions typical of a single nucleus.

The other way they tried to build in some coherence is in cutting of the cross sections for parton parton scattering at small angles. In a plasma, for example, such cross sections are cutoff by media screening effects. This parameter is crucial in their computations as all cross sections depend quadratically on such a cutoff.

In spite of these difficulties, the parton cascade model provides a useful way to describe the evolution of the matter from some time which I will refer to as the formation time $\tau_{f}$ until the thermalization time. The details of what is the precise form of the initial conditions and how one cuts off cross sections may be subject to dispute, but the description of the time evolution between $\tau_{f}$ and $\tau_{0}$ is conceptually correct.

There are several qualitative issues associated with the approach to equilibrium which we can easily understand from this approach. The first is that if the partons are formed in an energetic environment, then the coupling is weak. Thermalization will take place by two body scatterings. The number of quanta is conserved. Following the logic through the isentropic expansion stage, we see that the number of partons at formation is to a first approximation the same as the number of produced pions.

A second issue concerns flavor production. Initially most of the quanta are gluons. This is because they dominate the distribution functions. The number of quarks and anti-quarks in the sea is relatively small compared to the number of gluons. Therefore, the quark flavors come into chemical equilibrium during the transport and hydrodynamic evolution times, and therefore can be estimated by these methods if they turn out to be significantly in excess of their intrinsic contribution to the hadron wavefunction. 


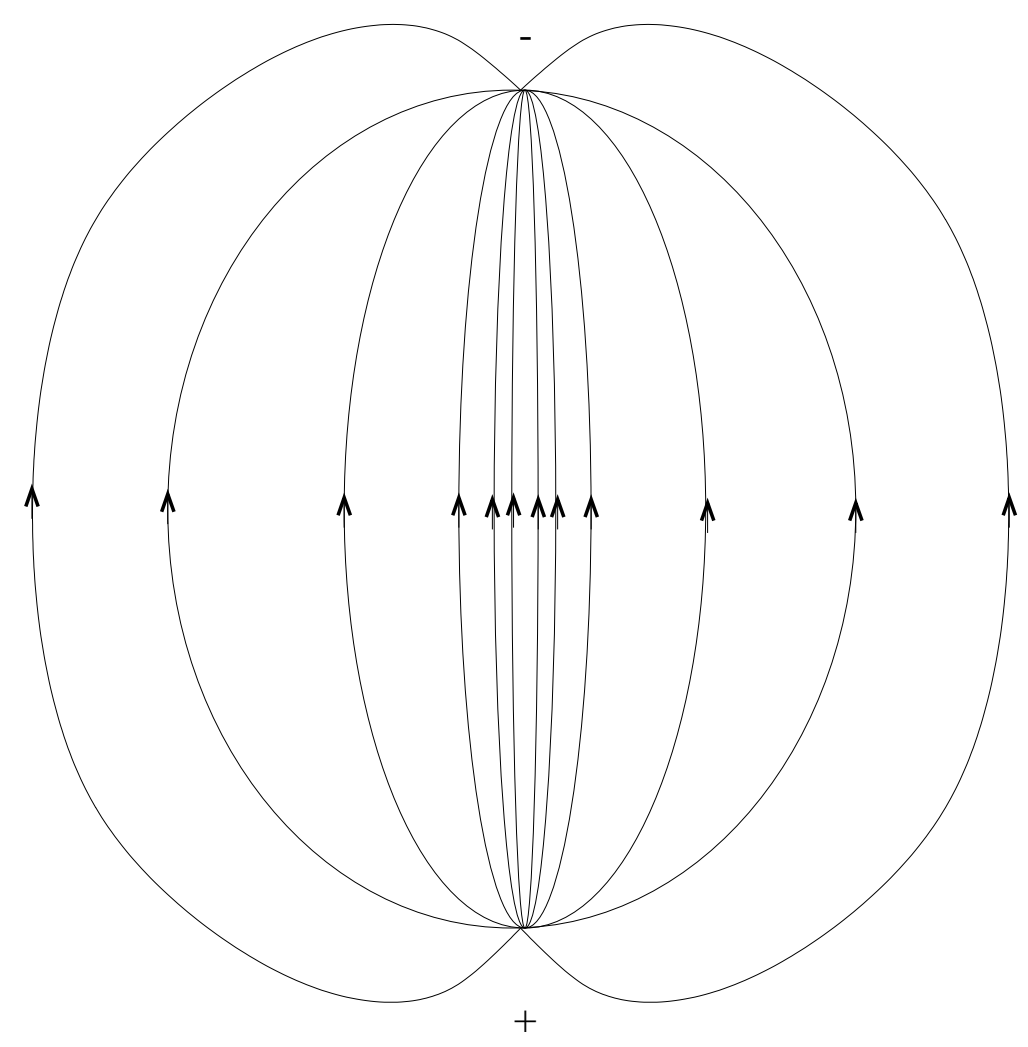

FIGURE 2. The Electric Field from a Dipole

\section{BEFORE THE PARTON CASCADE}

In order to poperly formulate the initial value conditions for the parton cascade, one must have a consistent quantum mechanical picture of the early stages of the collision. Such a picture is given by the McLerran-Venugopalan model as extended to nucleus-nucleus collisions. [4]-citekmw The basic ingredient in this picture are non-abelian Lienard-Wiechart potentials. To understand how this works, consider an electric dipole at rest. The electric field is the familiar electric field shown in Fig. 2.

If we now boost this field to the infinite momentum frame, the electric and magnetic fields all exist in a plane perpendicular to that of the direction of motion. Further, the magnetic field lines are perpendicular to the electric field lines. Viewed head on, the electric field lines are those of Fig. 2, with magnetic fields everywhere orthogonal to electric.

Now if we study the field produced at central rapidity by a fast moving nucleus, all the gluons at higher rapidity act as color sources for these fields. This means that the system is composed of very many dipole fields in an infinitesmally thin plane perpendicular to the direction of motion. Since color is confined, on scales larger than that of a fermi, the fields vanish. On smaller scales, they are stochastic. 
The McLerran-Venugopalan model assumes that these fields are generated by a Gaussian distribution of sources. The weight function for these distributions of sources may be directly related to the gluon distribution function.

The fields maintain their Lienard-Wiechart form prior to the collision. Upon collision, the fields begin evolving. The Yang-Mills equations can be solved numerically from these initial conditions. [7]-citebmp Initially, the fields are strong and the equations of motion are intrinsically non-linear. As the fields evolve, they dilute themselves and at some time the field equations linearize. The solution to the linear equation corresponds to produced gluons. One can compute their phase space density, and this forms the initial conditions for a subsequent cascade description.

There is only one scale in this classical problem: the total charge in gluon at rapidities other than the central region. Up to powers of $\alpha_{s}$, this is the same as the rapidity of gluon per unit area

$$
\Lambda^{2}=\frac{1}{\pi R^{2}} \frac{d N}{d y}
$$

If $\Lambda>>\Lambda_{Q C D}$, then the coupling at this scale is weak, and the classical description is consistent. (Factors of $\alpha_{s}$ can be ignored in the power counting arguments below as they involve only logarithms of density scales).

This single scale has many consequences. It is precisely the scale-introduced by hand in the parton cascade which is used to cutoff the parton cross sections. Note that it depends upon the initial density of partons per unit area, and therefore upon rapidity and the baryon number of the target. Verifying that there is in fact such a $p_{T}$ scale, and its dependence on various nuclei and rapidity will be one of the things that RHIC should be able to do.

Another consequence is because the density of produced gluon had the same parametric dependence on density as does the initial gluon density per unit rapidity, up to slowly varying factors of $\alpha_{s}$ and constant factors, these densities are the same. This provides an a posteriori justification for the initial conditions used in the parton cascade model. The space-time structure of the initial conditions is automatically built in to the classical computation. Several groups are now attempting solution of the classical field nucleus-nucleus collision problems, and this can provide an initialization for a parton cascade computation.

\section{ACKNOWLEDGMENTS}

I thank my colleagues Alejandro Ayala-Mercado, Miklos Gyulassy, Yuri Kovchegov, Alex Kovner, Jamal Jalilian-Marian, Andrei Leonidov, Raju Venugopalan and Heribert Weigert with whom the ideas presented in this talk were developed. This work was supported under Department of Energy grants in high energy and nuclear physics DOE-FG02-93ER-40764 and DOE-FG02-87-ER-40328. 


\section{REFERENCES}

1. K. Geiger and B. Muller, Nucl. Phys. B369 600 (1992).

2. K. Geiger, Phys. Rep., 258237 (1995).

3. J. D. Bjorken, Phys. Rev. D27 140 (1983).

4. L. McLerran and R. Venugopalan, Phys. Rev. D49 2233 (1994); D49 3352 (1994).

5. Y. Kovchegov, Phys. Rev. D54 5463 (1996); D55 5445 (1997).

6. A. Kovner, L. McLerran, and H. Weigert Phys. Rev. D52, 3809 (1995); 6231 (1995).

7. A. Krasnitz and R. Venugopalan, hep-ph/9809433

8. S. Bass, B. Muller and W. Poschl, nucl-ph/980804 